\title{
Analyticity and Global Existence of Small Solutions to Some Nonlinear Schrödinger Equations
}

\author{
Nakao Hayashi and Saburou Saitoh \\ Department of Mathematics, Faculty of Engineering, Gunma University, Kiryu 376, Japan
}

Abstract. In this paper we will study the nonlinear Schrödinger equations:

$$
\begin{aligned}
i \partial_{t} u+\frac{1}{2} \Delta u & =|u|^{2} u, \quad(t, x) \in \mathbb{R} \times \mathbb{R}_{x}^{n}, \\
u(0, x) & =\phi(x), \quad x \in \mathbb{R}_{x}^{n} .
\end{aligned}
$$

It is shown that the solutions of $(*)$ exist and are analytic in space variables for $t \in \mathbb{R} \backslash\{0\}$ if $\phi(x)\left(\in H^{2 n+1,2}\left(\mathbb{R}_{x}^{n}\right)\right)$ decay exponentially as $|x| \rightarrow \infty$ and $n \geqq 2$.

\section{Introduction and Results}

We consider the nonlinear Schrödinger equations

$$
\begin{aligned}
i \partial_{t} u+\frac{1}{2} \Delta u & =|u|^{2} u, \quad(t, x) \in \mathbb{R} \times \mathbb{R}_{x}^{n}, \\
u(0, x) & =\phi(x), \quad x \in \mathbb{R}_{x}^{n} .
\end{aligned}
$$

There are many works on the global existence of solutions to (1.1)-(1.2) in appropriate Sobolev spaces (see [2-5,8,10-13], and references cited therein). Furthermore it is known that (1.1)-(1.2) have a smoothing property that the solutions become smooth for $t \neq 0$ even if their initial data are not smooth. More precisely, in [7] it was shown that all solutions of (1.1)-(1.2) are smooth for $t \neq 0$ provided that the initial data in $H^{[n / 2]+1,2}\left(\mathbb{R}_{x}^{n}\right)$ decay sufficiently rapidly as $|x| \rightarrow \infty$. Our aim of this paper is twofold. One is to show that if the initial data $\phi$ are analytic and sufficiently small in an appropriate norm, then the solutions of (1.1)-(1.2) exist globally in time and are analytic in space variables. The other is to show that if the initial data $\phi$ in $H^{2 n+1,2}\left(\mathbb{R}_{x}^{n}\right)$ decay exponentially as $|x| \rightarrow \infty$ and are sufficiently small in an appropriate norm, then the solutions of (1.1)-(1.2) exist globally in time and are analytic in space variables for $t \in \mathbb{R} \backslash\{0\}$.

We give notation and function spaces used in this paper.

Notation and Function Spaces. We let $L^{p}\left(\mathbb{R}_{x}^{n}\right)=\left\{f(x) ; f(x)\right.$ is measurable on $\mathbb{R}_{x}^{n}$, $\left.|f(x)|_{L^{p}\left(\mathbb{R}_{x}^{n}\right)}<\infty\right\}$, where $|f(x)|_{L^{p}\left(\mathbb{R}_{x}^{n}\right)}=\left(\int_{\mathbb{R}_{x}^{n}}|f(x)|^{p} d x\right)^{1 / p}$ if $1 \leqq p<\infty$ and $|f(x)|_{L^{\infty}\left(\mathbb{R}_{x}^{n}\right)}=$ 
ess.sup $\left\{|f(x)| ; x \in \mathbb{R}_{x}^{n}\right\}$ if $p=\infty$, and we let $H^{m, p}\left(\mathbb{R}_{x}^{n}\right)=\left\{f(x) \in L^{p}\left(\mathbb{R}_{x}^{n}\right) ;|f(x)|_{H^{m, p}\left(\mathbb{R}_{x}^{n}\right)}=\right.$ $\left.\left|\left[\mathscr{F}^{-1}\left(1+|\xi|^{2}\right)^{m / 2} \mathscr{F} f\right](x)\right|_{L^{p}\left(\mathbb{R}_{x}^{n}\right)}<\infty\right\}$, where $m \in \mathbb{R}^{+},|\xi|^{2}=\sum_{j=1}^{n} \xi_{j}^{2}, \quad[\mathscr{F} f](\xi)=$ $\int e^{-i x \xi} f(x) d x$ (or $\left.\hat{f}(\xi)\right)$ is the Fourier transform of $f(x)$ and $\left[\mathscr{F}^{-1} g\right](x)=$ $\mathbb{R}_{x}^{n}$

$\frac{1}{(2 \pi)^{n}} \int_{\mathbb{R}_{x}^{n}} e^{i x \xi} g(\xi) d \xi$ is the inverse Fourier transform of $g(\xi)$, respectively. We denote the $L^{2}\left(\mathbb{R}_{x}^{n}\right)$-scalar product by $(\cdot, \cdot)$. For each $r>0$ we denote by $S(r)$ the strip $\left\{-r<\operatorname{Im} z_{j}<r ; 1 \leqq j \leqq n\right\}$ in $\mathbb{C}^{n}$. For $x \in \mathbb{R}_{x}^{n}$, if a complex-valued function $f(x)$ has an analytic continuation to $S(r)$, then we denote this by the same letter $f(z)$, and if $g(z)$ is an analytic function on $S(r)$, then we denote the restriction of $g(z)$ to the real axis by $g(x)$. We let

$$
A L^{p}(r)=\left\{f(z) ; f(z) \text { is analytic on } S(r),|f(z)|_{A L^{p}(r)}<\infty\right\},
$$

where

$$
\begin{aligned}
|f(z)|_{A L^{p}(r)}^{p} & =\frac{1}{(2 r)^{n}} \int_{(-r, r)^{n}} \int_{\mathbb{R}_{x}^{n}}|f(z)|^{p} d x d y \\
& =\frac{1}{(2 r)^{n}} \int_{S(r)}|f(z)|^{p} d x d y \quad \text { if } \quad 1 \leqq p<\infty
\end{aligned}
$$

and

$$
|f(z)|_{A L^{x}(r)}=\sup \{|f(z)| ; z \in S(r)\} \quad \text { if } \quad p=\infty
$$

and we let

$$
A H^{m, p}(r)=\left\{f(z) \in A L^{p}(r) ;|f(z)|_{A H^{m, p}(r)}=\left|\left[\mathscr{F}^{-1}\left(1+|\xi|^{2}\right)^{m / 2} \mathscr{F} f\right](z)\right|_{A L^{p}(r)}<\infty\right\},
$$

where $m \in \mathbb{R}^{+}$. We note that if $m \in \mathbb{N}$, then

$$
H^{m, p}\left(\mathbb{R}_{x}^{n}\right)=\left\{f(x) \in L^{p}\left(\mathbb{R}_{x}^{n}\right) ;|f(x)|_{\left.H^{m, p_{(}} \mathbb{R}_{x}^{n}\right)}=\sum_{|\alpha| \leqq m}\left|\partial_{x}^{\alpha} f(x)\right|_{L^{p}\left(\mathbb{R}_{x}^{n}\right)}<\infty\right\}
$$

and

$$
A H^{m, p}(r)=\left\{f(z) \in A L^{p}(r) ;|f(z)|_{A H^{m, p}(r)}=\sum_{|\alpha| \leqq m}\left|\partial_{x}^{\alpha} f(z)\right|_{A L^{p}(r)}=\sum_{|\alpha| \leqq m}\left|\partial_{z}^{\alpha} f(z)\right|_{A L^{p}(r)}<\infty\right\},
$$

where $\alpha=\left(\alpha_{1}, \ldots, \alpha_{n}\right) \in(\mathbb{N} \cup\{0\})^{n}$ is a multi-index, $\partial_{x}^{\alpha}=\partial_{x_{1}}^{\alpha_{1}} \cdots \partial_{x_{n}}^{\alpha_{n}}, \partial_{z}^{\alpha}=\partial_{z_{1}}^{\alpha_{1}} \cdots \partial_{z_{n}}^{\alpha_{n}}$, and $|\alpha|=\alpha_{1}+\cdots+\alpha_{n}$. For any interval $I$ of $\mathbb{R}$ and any Banach space $B$ with the norm $|\cdot|_{B}$, we let $C(I ; B)=\left\{f(t) ; f(t)\right.$ is continuous from $I$ to $\left.B, \sup \left\{|f(t)|_{B} ; t \in I\right\}<\infty\right\}$, $C^{m}(I ; B)=\left\{f(t) \in C(I ; B) ; \sup \left\{\sum_{j=0}^{m}\left|\partial_{t}^{j} f(t)\right|_{B} ; t \in I\right\}<\infty\right\}$. Constants will be denoted by $C_{j}(j=1,2, \ldots)$. If necessary, by $C_{j}(*, \ldots, *)$ we denote constants depending only on the quantities appearing in parentheses.

We now state our results in this paper.

Theorem 1. We assume that $\phi(x)$ has an analytic continuation to $S(r)$ and $M=\sum_{|\alpha|+|\beta| \leqq 2 n+1}\left|\partial_{z}^{\alpha} z^{\beta} \phi(z)\right|_{A L^{2}(r)}^{2}$ is sufficiently small, where $z^{\beta}=z_{1}^{\beta_{1}} \cdots z_{n}^{\beta_{n}}$ and $n \geqq 2$. 
Then, there exists a unique global solution $u(t, x)$ of $(1.1)-(1.2)$ such that $u(t, x)$ has an analytic continuation to $S(r)$ and

$$
u(t, z) \in C^{1}\left(\mathbb{R} ; A H^{2 n-1,2}(r)\right) \cap C\left(\mathbb{R} ; A H^{2 n+1,2}(r)\right) .
$$

Furthermore, $u(t, z)$ satisfies

$$
\sum_{|\alpha|+|\beta| \leqq 2 n+1}\left|\partial_{z}^{\alpha} J_{z}^{\beta} u(t, z)\right|_{A L^{2}(r)}^{2} \leqq C_{1}(M)
$$

where

$$
J_{z}^{\beta}=J_{z_{1}}^{\beta_{1}} \cdots J_{z_{n}}^{\beta_{n}}, \quad J_{z_{j}}=e^{i|z|^{2} / 2 t} i t \partial_{z_{j}} e^{-i|z|^{2} / 2 t}=z_{j}+i t \partial_{z_{j}}, \quad|z|^{2}=\sum_{j=1}^{n} z_{j}^{2} .
$$

Theorem 2. We assume that $[\mathscr{F} \phi](\xi)$ has an analytic continuation to $S(1)$ and $N=\sum_{|\alpha|+|\beta| \leqq 2 n+1}\left|\partial_{z}^{\alpha} z^{\beta}[\mathscr{F} \phi](z)\right|_{A L^{2}(1)}^{2}$ is sufficiently small, where $n \geqq 2$. Then there exists a unique global solution $u(t, x)$ of (1.1)-(1.2) such that

$$
u(t, x) \in C^{1}\left(\mathbb{R} ; H^{2 n-1,2}\left(\mathbb{R}_{x}^{n}\right)\right) \cap C\left(\mathbb{R} ; H^{2 n+1.2}\left(\mathbb{R}_{x}^{n}\right)\right),
$$

$e^{i t|\xi|^{2} / 2}[\mathscr{F} u](t, \xi)$ has an analytic continuation to $S(1)$ and

$$
e^{i t|z|^{2} / 2}[\mathscr{F} u](t, z) \in C\left(\mathbb{R} ; A H^{2 n+1.2}(1)\right) .
$$

Furthermore, $e^{i t|z|^{2} / 2}[\mathscr{F} u](t, z)$ satisfies

$$
\sum_{|\alpha|+|\beta| \leqq 2 n+1}\left|\partial_{z}^{\alpha} z^{\beta} e^{i t|z|^{2} / 2}[\mathscr{F} u](t, z)\right|_{A L^{2}(1)}^{2} \leqq C_{2}(N) .
$$

The paper was inspired by the work of T. Kato and K. Masuda [9]. In [9], they proved that if the initial function of the Korteweg-de Vries or the Benjamin-Ono equation has an analytic continuation to $S(r)$ and is in $A L^{2}(r)$, then the solution has an analytic continuation to $S\left(r_{1}\right)$ and is in $A L^{2}\left(r_{1}\right)$ for some time interval which depends on the size of the initial function, where $r>r_{1}$. They obtained the above result by making use of a class of Liapunov functions involving a parameter. Their method is useful to prove the analyticity of solutions of another type equations such as the Euler equation, the Navier-Stokes equation, nonlinear Schrödinger equation, etc. Thus our result of Theorem 1 is similar to the result of T. Kato and K. Masuda. However, their result is different from ours, since their result follows from Theorem 1 immediately in the case of (1.1)-(1.2). In fact, $\phi \in A L^{2}(r)$ implies $\phi \in A H^{m, 2}\left(r_{1}\right)$ for any $m \in \mathbb{N}$ provided that $r_{1}<r$, therefore by Theorem 1 we easily see that the solution of (1.1)-(1.2) is in $A H^{m, 2}\left(r_{1}\right)$ if $\phi \in A L^{2}(r)$. Moreover, Theorem 2 does not seem to be obtainable by their method which requires the condition $r_{1}<r$.

In [13], J. C. H. Simon and E. Taflin obtained the global existence theorem of analytic solutions for more general nonlinear Schrödinger equations than ours in three space dimensions. They proved that the solutions are analytic in a neighbourhood of $\mathbb{R}^{+} \times \mathbb{R}_{x}^{3}$ under the conditions on the initial data $\phi(x)$ such that the support of $\hat{\phi}(\xi)$ is contained in $\left\{\xi \in \mathbb{R}_{\xi}^{3} ;(a+1)^{-1} \leqq|\xi| \leqq a\right\}$ and $\hat{\phi}(\xi) \in C^{14}\left(\mathbb{R}_{\xi}^{3}\right)$. 
We note that their conditions on the initial data $\phi$ are stronger than ours (see Theorem 1).

Our results are based on the following

Lemma 1. If $f(z) \in A H^{m, 2}(r)$, then we have

$$
|f(z)|_{A H^{m, 2}(r)}^{2}=\sum_{\gamma} \frac{(2 r)^{2|\gamma|}}{(2 \gamma+1) !}\left|\partial_{x}^{\gamma}\left[\mathscr{F}^{-1}\left(1+|\xi|^{2}\right)^{m / 2} \mathscr{F} f\right](x)\right|_{L^{2}\left(\mathbb{R}_{x}^{n}\right)}^{2},
$$

where $\gamma$ is a multi-index, $|\gamma|=\gamma_{1}+\cdots+\gamma_{n},(2 \gamma+1) !=\left(2 \gamma_{1}+1\right) ! \cdots\left(2 \gamma_{n}+1\right) !$, and $\sum_{\gamma}=\sum_{\gamma \in(\mathbb{N} \cup\{0\})^{n}}$. Conversely, for a function $f(x)$ with a finite integral (1.8) on the real lines, there exists an analytic extension $\tilde{f}(z)$ such that $f(x)=\tilde{f}(x)$ a.e. on $\mathbb{R}^{n}$ and $\tilde{f}(z) \in A H^{m, 2}(r)$ satisfying the identity (1.8).

Proof. Lemma 1 was already obtained in [6] in the case of one space dimension. In the case of higher dimensional space, Lemma 1 is also obtained in the same way as in the proof of one space dimension, and so we omit it (see Lemma 1 in [6] for details).

We give some remarks on Theorem 2.

Remark 1. From Lemma 1, we have easily

$$
\begin{aligned}
\sum_{|\alpha|+|\beta| \leqq 2 n+1}\left|\partial_{z}^{\alpha} z^{\beta} \hat{\phi}(z)\right|_{A L^{2}(1)}^{2} & =\sum_{|\alpha|+|\beta| \leqq 2 n+1} \sum_{\gamma} \frac{2^{2|\gamma|}}{(2 \gamma+1) !}\left|\partial_{\xi}^{\gamma+\alpha} \xi^{\beta} \hat{\phi}(\xi)\right|_{L^{2}\left(\mathbb{R}_{\xi}^{n}\right)}^{2} \\
& =\sum_{|\alpha|+|\beta| \leqq 2 n+1} \sum_{\gamma} \frac{2^{2|\gamma|}}{(2 \gamma+1) !}\left|x^{\gamma+\alpha} \partial_{x}^{\beta} \phi(x)\right|_{L^{2}\left(\mathbb{R}_{x}^{n}\right)}^{2} \\
& =\sum_{|\alpha|+|\beta| \leqq 2 n+1} \int_{\mathbb{R}_{x}^{n}} \prod_{j=1}^{n} \frac{\sinh 2 x_{j}}{2 x_{j}}\left|x^{\alpha} \partial_{x}^{\beta} \phi(x)\right|^{2} d x .
\end{aligned}
$$

Thus the assumption of Theorem 2 is satisfied if the right-hand side of (1.9) is sufficiently small. Namely, Theorem 2 is valid if the initial data $\phi$ in $H^{2 n+1,2}\left(\mathbb{R}_{x}^{n}\right)$ decay exponentially as $|x| \rightarrow \infty$ and are sufficiently small in the sense of the norm (1.9).

Remark 2. From Lemma $1, e^{i t|z|^{2} / 2} \hat{u}(t, z) \in A L^{2}(1)$ shows that

$$
\begin{aligned}
\left|e^{i t|z|^{2} / 2} \hat{u}(t, z)\right|_{A L^{2}(1)}^{2} & =\sum_{\gamma} \frac{2^{2|\gamma|}}{(2 \gamma+1) !}\left|\partial_{\xi}^{\gamma} e^{i t|\xi|^{2} / 2} \hat{u}(t, \xi)\right|_{L^{2}\left(\mathbb{R}_{\xi}^{n}\right)}^{2} \\
& =\sum_{\gamma} \frac{2^{2|\gamma|}}{(2 \gamma+1) !}\left|x^{\gamma}\left[\mathscr{F}^{-1} e^{i|\xi|^{2} t / 2} \mathscr{F} u\right](t, x)\right|_{L^{2}\left(\mathbb{R}_{x}^{n}\right)}^{2} \\
& =\sum_{\gamma} \frac{2^{2|\gamma|}}{(2 \gamma+1) !}\left|x^{\gamma} U(-t) u(t, x)\right|_{L^{2}\left(\mathbb{R}_{x}^{n}\right)}^{2} \\
& =\sum_{\gamma} \frac{(2|t|)^{2|\gamma|}}{(2 \gamma+1) !}\left|\partial_{x}^{\gamma} e^{-i|x| 2 / 2 t} u(t, x)\right|_{L^{2}\left(\mathbb{R}_{x}^{n}\right)}^{2} \\
& =\left|e^{-i|z|^{2} / 2 t} u(t, z)\right|_{A L^{2}(|t|)}^{2},
\end{aligned}
$$


where $U(t)$ is the evolution operator associated with free Schrödinger equations, and we have used the fact that $U(t) x^{\gamma} U(-t)=e^{-i|x|^{2} / 2 t}\left(i t \partial_{x}\right)^{\gamma} e^{i|x|^{2} / 2 t}$. Thus Theorem 2 implies that the solution of (1.1)-(1.2) is analytic in space variables if $t \in \mathbb{R} \backslash\{0\}$.

Remark 3. Similar result of Theorem 2 has been obtained in [1] in the case of semi-linear heat equations.

The paper is organized as follows. In Sect. 2 we prove the theorems. In Sect. 3 we give some lemmas which are needed for that purpose.

\section{Proofs of Theorems 1-2}

We will prove our theorems by making use of the contraction mapping principle in function spaces stated below.

Proof of Theorem 1. For any $r>0$, we define the Banach space $B_{1}(\mathbb{R})$ by

$$
B_{1}(\mathbb{R})=\left\{v(t, z) \in C\left(\mathbb{R} ; A L^{2}(r)\right) ;|v(z)|_{B_{1}(\mathbb{R})}=\sup \left\{|v(z)|_{B_{1}(t)} ; t \in \mathbb{R}\right\}<\infty\right\},
$$

where $|v(z)|_{B_{1}(t)}^{2}=\sum_{|\beta|+|\beta| \leqq 2 n+1}\left|\partial_{z}^{\alpha} J_{z}^{\beta} v(t, z)\right|_{A L^{2}(r)}^{2}$. For any $v(t, z) \in B_{1}(\mathbb{R})$ we define the operator $M$ by

$$
M v(t, x)=U(t) \phi(x)-i \int_{0}^{t} U(t-s) v(s, x)^{2} \bar{v}(s, x) d s .
$$

We denote by $B_{1}^{\rho}(\mathbb{R})$ the closed ball in $B_{1}(\mathbb{R})$ with radius $\rho>0$, where $\rho=2\left(\sum_{|\alpha|+|\beta| \leqq 2 n+1}\left|\partial^{\alpha} z^{\beta} \phi(z)\right|_{A L^{2}(r)}\right)^{1 / 2}$. We will prove that if $\rho$ is sufficiently small, then the mapping $M$ is a contraction map from $B_{1}^{\rho}(\mathbb{R})$ into itself. We only treat the case $t>0$, since $t<0$ can be treated similarly. We put $u=M v$, then $u$ satisfies

$$
\begin{gathered}
i \partial_{t} u+\frac{1}{2} \Delta u=v^{2} \bar{v}, \quad(t, x) \in \mathbb{R}^{+} \times \mathbb{R}_{x}^{n}, \\
u(0, x)=\phi(x), \quad x \in \mathbb{R}_{x}^{n} .
\end{gathered}
$$

We apply $\partial_{x}^{\gamma+\alpha} J_{x}^{\beta}$ to Eq. (2.1) to obtain

$$
i \partial_{t} \partial_{x}^{\gamma+\alpha} J_{x}^{\beta} u+\frac{1}{2} \Delta \partial_{x}^{\gamma+\alpha} J_{x}^{\beta} u=\partial_{x}^{\gamma+\alpha} J_{x}^{\beta} v^{2} \bar{v}
$$

since $\left[i \partial_{t}+\frac{1}{2} \Delta, \partial_{x}^{\gamma+\alpha} J_{x}^{\beta}\right]=0$. Multiplying both sides of $(2.3)$ by $\left((2 r)^{2|\gamma|}\right.$ $(2 \gamma+1) !) \partial_{x}^{\gamma+\alpha} \bar{J}_{x}^{\beta} \bar{u}$, integrating in $x$ and taking the imaginary part, we have

$$
\begin{aligned}
\frac{1}{2} & \frac{d}{d t} \sum_{|\gamma| \leqq m} \frac{(2 r)^{2|\gamma|}}{(2 \gamma+1) !}\left|\partial_{x}^{\gamma+\alpha} J_{x}^{\beta} u(t, x)\right|_{L^{2}\left(\mathbb{R}_{x}^{n}\right)}^{2} \\
= & \operatorname{Im} \sum_{|\gamma| \leqq m} \frac{(2 r)^{2|\gamma|}}{(2 \gamma+1) !}\left(\partial_{x}^{\gamma+\alpha} J_{x}^{\beta} v^{2} \bar{v}(t, x), \partial_{x}^{\gamma+\alpha} J_{x}^{\beta} u(t, x)\right) \\
\leqq & \left(\sum_{|\gamma| \leqq m} \frac{(2 r)^{2|\gamma|}}{(2 \gamma+1) !}\left|\partial_{x}^{\gamma+\alpha} J_{x}^{\beta} v^{2} \bar{v}(t, x)\right|_{L^{2}\left(\mathbb{R}_{x}^{n}\right)}^{2}\right)^{1 / 2} \\
& \cdot\left(\sum_{|\gamma| \leqq m} \frac{(2 r)^{2|\gamma|}}{(2 \gamma+1) !}\left|\partial_{x}^{\gamma+\alpha} J_{x}^{\beta} u(t, x)\right|_{L^{2}\left(\mathbb{R}_{x}^{n}\right)}^{2}\right)^{1 / 2}
\end{aligned}
$$


where we have used the Schwarz inequality. From (2.4)

$$
\begin{aligned}
& \left(\sum_{|\gamma| \leqq m} \frac{(2 r)^{2|\gamma|}}{(2 \gamma+1) !}\left|\partial_{x}^{\gamma+\alpha} J_{x}^{\beta} u(t, x)\right|_{L^{2}\left(\mathbb{R}_{x}^{n}\right)}^{2}\right)^{1 / 2} \\
& \leqq\left(\sum_{|\gamma| \leqq m} \frac{(2 r)^{2|\gamma|}}{(2 \gamma+1) !}\left|\partial_{x}^{\gamma+\alpha} x^{\beta} \phi(x)\right|_{L^{2}\left(\mathbb{R}_{x}^{n}\right)}^{2}\right)^{1 / 2} \\
& \quad+\int_{0}^{t}\left(\sum_{|\gamma| \leqq m} \frac{(2 r)^{2|\gamma|}}{(2 \gamma+1) !}\left|\partial_{x}^{\gamma+\alpha} J_{x}^{\beta} v^{2} \bar{v}(s, x)\right|_{L^{2}\left(\mathbb{R}_{x}^{n}\right)}^{2}\right)^{1 / 2} d s .
\end{aligned}
$$

We let $m$ tend to infinity and use Lemma 1 to obtain

$$
\left|\partial_{z}^{\alpha} J_{z}^{\beta} u(t, z)\right|_{A L^{2}(r)} \leqq\left|\partial_{z}^{\alpha} z^{\beta} \phi(z)\right|_{A L^{2}(r)}+\int_{0}^{t}\left|\partial_{z}^{\alpha} J_{z}^{\beta} v^{2}(s, z) \overline{v(s, \bar{z})}\right|_{A L^{2}(r)} d s .
$$

From this it follows that

$$
|u(z)|_{B_{1}(t)} \leqq \sum_{|\alpha|+|\beta| \leqq 2 n+1}\left|\partial_{z}^{\alpha} z^{\beta} \phi(z)\right|_{A L^{2}(r)}+\int_{0}^{t}\left|v^{2}(z) \overline{v(\bar{z})}\right|_{B_{1}(s)} d s .
$$

Applying Lemma 3.2 ((3.21)) to (2.5), we have

$$
\begin{aligned}
|u(z)|_{B_{1}(t)} & \leqq \frac{\rho}{2}+C_{3} \int_{0}^{t}(1+s)^{-n}|v(z)|_{B_{1}(s)}^{3} d s \\
& \leqq \frac{\rho}{2}+C_{3} \cdot \rho^{3} \int_{0}^{t}(1+s)^{-n} d s .
\end{aligned}
$$

From this we have

$$
|u(z)|_{B_{1}(\mathbb{R})} \leqq \frac{\rho}{2}+C_{4} \cdot \rho^{3}
$$

Let $v_{j}(t, z) \in B_{1}^{\rho}(\mathbb{R})(j=1,2)$ and $u_{j}=M v_{j}$ be the solution of

$$
\begin{gathered}
i \partial_{t} u_{j}+\frac{1}{2} \Delta u_{j}=v_{j}^{2} \bar{v}_{j}, \quad(t, x) \in \mathbb{R}^{+} \times \mathbb{R}_{x}^{n}, \\
u_{j}(0, x)=\phi(x), \quad x \in \mathbb{R}_{x}^{n} .
\end{gathered}
$$

In the same way as in the proof of (2.5),

$$
\left|u_{1}(z)-u_{2}(z)\right|_{B_{1}(t)} \leqq \int_{0}^{t}\left|v_{1}^{2}(z) \overline{v_{1}(\bar{z})}-v_{2}^{2}(z) \overline{v_{2}(\bar{z})}\right|_{B_{1}(s)} d s .
$$

We apply Lemma $3.2((3.22))$ to (2.9) to obtain

$$
\begin{aligned}
\left|u_{1}(z)-u_{2}(z)\right|_{B_{1}(\mathbb{R})} \leqq & C_{5} \cdot\left(\left|v_{1}(z)\right|_{B_{1}(\mathbb{R})}^{2}+\left|v_{2}(z)\right|_{B_{1}(\mathbb{R})}^{2}\right) \\
& \cdot \int_{0}^{t}(1+s)^{-n}\left|v_{1}(z)-v_{2}(z)\right|_{B_{1}(s)} d s \\
\leqq & C_{6} \rho^{2}\left|v_{1}(z)-v_{2}(z)\right|_{B_{1}(\mathbb{R})} .
\end{aligned}
$$


If $\rho$ is sufficiently small, then (2.6) and (2.10) imply that $M$ is a contraction map from $B_{1}^{\rho}(\mathbb{R})$ into itself. This implies that there exists a unique global solution of (1.1)-(1.2) satisfying (1.4). From (1.4) and (1.2) we have easily (1.3). Q.E.D.

Proof of Theorem 2. We define the Banach space $B_{2}(\mathbb{R})$ by

$$
B_{2}(\mathbb{R})=\left\{v(t, x) \in C\left(\mathbb{R} ; L^{2}\left(\mathbb{R}^{n}\right)\right) ;|v(z)|_{B_{2}(\mathbb{R})}=\sup \left\{|v(z)|_{B_{2}(t)} ; t \in \mathbb{R}\right\}<\infty\right\},
$$

where

$$
|v(z)|_{B_{2(t)}}^{2}=\sum_{|\alpha|+|\beta| \leqq 2 n+1}\left|\partial_{z}^{\alpha} e^{i t|z|^{2} / 2} z^{\beta} \hat{v}(t, z)\right|_{A L^{2}(1)}^{2} .
$$

We denote by $B_{2}^{\rho}(\mathbb{R})$ the closed ball in $B_{2}(\mathbb{R})$ with radius $\rho>0$, where $\rho=2\left(\sum_{|\alpha|+|\beta| \leqq 2 n+1}\left|\partial_{z}^{\alpha} z^{\beta} \hat{\phi}(z)\right|_{A L^{2}(1)}\right)^{1 / 2}$. We let $v_{j}(t, z) \in B_{2}^{\rho}(\mathbb{R})(j=1,2)$ and consider the Schrödinger equations (2.7)-(2.8). By the Fourier transform we have

$$
\begin{aligned}
i \partial_{t} \hat{u}_{j}(t, \xi)-\frac{1}{2}|\xi|^{2} \hat{u}_{j}(t, \xi) & =\left[\mathscr{F} v_{j}^{2} \bar{v}_{j}\right](t, \xi), \quad(t, \xi) \in \mathbb{R}^{+} \times \mathbb{R}_{\xi}^{n}, \\
\hat{u}_{j}(0, \xi) & =\hat{\phi}(\xi), \quad \xi \in \mathbb{R}_{\xi}^{n},
\end{aligned}
$$

from which it follows that

$$
\begin{gathered}
i \partial_{t} e^{i t|\xi|^{2} / 2} \hat{u}_{j}(t, \xi)=e^{i t|\xi|^{2} / 2}\left[\mathscr{F} v_{j}^{2} \bar{v}_{j}\right](t, \xi), \\
\hat{u}_{j}(0, \xi)=\hat{\phi}(\xi) .
\end{gathered}
$$

In the same way as in the proof of (2.6) and (2.10), we have by (2.11), (2.12) and Lemma $3.2\left(\left(3.21^{\prime}\right)-\left(3.22^{\prime}\right)\right)$

$$
\begin{aligned}
\left|u_{j}(z)\right|_{B_{2}(\mathbb{R})} \leqq \frac{\rho}{2}+C_{7} \rho^{3}, \quad(j=1,2), & \\
\left|u_{1}(z)-u_{2}(z)\right|_{B_{2}(\mathbb{R})} & \leqq C_{8} \rho^{2}\left|v_{1}(z)-v_{2}(z)\right|_{B_{2}(\mathbb{R})} .
\end{aligned}
$$

The above inequalities show that $M$ is a contraction map from $B_{2}^{\rho}(\mathbb{R})$ into itself provided that $\rho$ is sufficiently small. This implies that there exists a unique global solution of (1.1)-(1.2) satisfying (1.5), (1.6) and (1.7). Q.E.D.

\section{Lemmas}

In this section we use the following notation:

$$
\begin{aligned}
P\left(2 i r \partial_{x}\right) & =\prod_{j=1}^{n}\left(1+\left(2 i r \partial_{x_{j}}\right)^{2}\right)^{1 / 4}=\mathscr{F}^{-1} \prod_{j=1}^{n}\left(1+\left(2 r \xi_{j}\right)^{2}\right)^{1 / 4} \mathscr{F}, \\
P(2 x) & =\prod_{j=1}^{n}\left(1+\left(2 x_{j}\right)^{2}\right)^{1 / 4}, \quad b=\frac{n}{2} /\left(\left[\frac{n}{2}\right]+1\right), \\
Q(a, r) & =\frac{(2 r)^{2 a}}{(2 a+1) !} \text { for } \quad a \in \mathbb{N} \cup\{0\},
\end{aligned}
$$


and

$$
Q(\gamma, r)=\frac{(2 r)^{2|\gamma|}}{(2 \gamma+1) !} \text { for a multi-index } \gamma
$$

where $[s]$ denotes the largest integer that is not larger than $s$.

We first derive several $L^{\infty}$ estimates for analytic functions which are proved by using the Gagliardo-Nirenberg-Sobolev inequality and the $L^{\infty}$ decay estimate of the evolution operator associated with free Schrödinger equations.

Lemma 3.1. 1. Let $\partial_{z}^{\alpha} f(z) \in A H^{n+1,2}(r)$. Then we have

$$
\begin{aligned}
\left|\partial_{z}^{\alpha} f(z)\right|_{A L^{x}(r)} \leqq & C_{9}\left|P\left(2 i r \partial_{x}\right) \partial_{z}^{\alpha} f(z)\right|_{A L^{2}(r)}^{1-b} \\
& \cdot\left(\sum_{|\beta|=[n / 2]+1}\left|P\left(2 i r \partial_{x}\right) \partial_{z}^{\alpha+\beta} f(z)\right|_{A L^{2}(r)}^{2}\right)^{b / 2} .
\end{aligned}
$$

2. Let $\partial_{z}^{\alpha-v} J_{z}^{\beta-\delta-v} f(z) \in A H^{n / 2,2}(r)$, for $v \leqq \min (\beta-\delta, \alpha), \delta \leqq \beta$. Here, $v \leqq \min (\beta-\delta, \alpha)$ and $\delta \leqq \beta$ mean that $v_{j} \leqq \min \left(\beta_{j}-\delta_{j}, \alpha_{j}\right)$ and $\delta_{j} \leqq \beta_{j}(1 \leqq j \leqq n)$, respectively. Then we have

$$
\begin{aligned}
\left|\partial_{z}^{\alpha} f(z)\right|_{A L^{\infty}(r)} \leqq & C_{10}(\alpha)|t|^{-n / 2}\left|P\left(2 i r \partial_{x}\right) \partial_{z}^{\alpha} f(z)\right|_{A L^{2}(r)}^{1-b} \\
& \cdot \sum_{\substack{\delta \leqq \beta \\
|\beta|=[n / 2]+1}} \sum_{v \leqq \min (\beta-\delta, \alpha)}\left\{\begin{array}{l}
\left(r^{2|\delta|}\left|P\left(2 i r \partial_{x}\right) \partial_{z}^{\alpha-v} J_{z}^{\beta-\delta-v} f(z)\right|_{A L^{2}(r)}^{2}\right)^{b / 2} \\
\left(r^{2|\delta|}\left|P\left(2 i r \partial_{x}\right) \partial_{z}^{\alpha-v} \bar{J}_{\bar{z}}^{\beta-\delta-v} f(z)\right|_{A L^{2}(r)}\right)^{b / 2}
\end{array}\right.
\end{aligned}
$$

3. We assume that $e^{i t|\xi|^{2} / 2} \hat{g}(\xi)$ has an analytic continuation to $S(1)$ and $\partial_{z}^{\alpha} e^{i t|z|^{2} / 2} \hat{g}(z)$ $\in A H^{n+1,2}(1)$. Then we have

$$
\begin{aligned}
\left|\left(i t \partial_{z}\right)^{\alpha} e^{-i|z|^{2} / 2 t} g(z)\right|_{A L^{\infty}(|t|)} \leqq & C_{9}|t|^{-n / 2} \mid P\left(2 i \partial_{x}\right) \partial_{z}^{\alpha} e^{i t|z|^{2} / 2} \hat{g}(z)_{A L^{2}(1)}^{1-b} \\
& \cdot\left(\sum_{|\beta|=[n / 2]+1}\left|P\left(2 i \partial_{x}\right) \partial_{z}^{\alpha+\beta} e^{i t|z|^{2} / 2} \hat{g}(z)\right|_{A L^{2}(1)}^{2}\right)^{b / 2} .
\end{aligned}
$$

4. We assume that $e^{i t|\xi|^{2 / 2} \xi^{\beta-\delta-v}} \hat{g}(\xi)$ has an analytic continuation to $S(1)$ and $\partial_{z}^{\alpha-v} e^{i t|z|^{2} / 2} z^{\beta-\delta-v} \hat{g}(z) \in A H^{n / 2,2}(1)$, where $v \leqq \min (\beta-v, \alpha), \delta \leqq \beta$. Then we have

$$
\begin{aligned}
& \left|\left(i t \partial_{z}\right)^{\alpha} e^{-i|z|^{2} / 2 t} g(z)\right|_{A L^{x}(|t|)} \\
& \leq C_{10}(\alpha)\left|P\left(2 i \partial_{x}\right) \partial_{z}^{\alpha} e^{i t|z|^{2} / 2} \hat{g}(z)\right|_{A L^{2}(1)}^{1-b} \\
& \quad \cdot \sum_{\substack{\delta \leqq \beta \\
|\beta|=[n / 2]+1}} \sum_{v \leqq \min (\beta-\delta, \alpha)}\left(\left|P\left(2 i \partial_{x}\right) \partial_{z}^{\alpha-v} e^{i t|z|^{2} / 2} z^{\beta-\delta-v} \hat{g}(z)\right|_{A L^{2}(1)}^{2}\right)^{b / 2}
\end{aligned}
$$

Proof. By Sobolev's inequality we have with $m=[n / 2]+1$,

$$
\left|\partial_{z}^{\alpha} f(z)\right|_{L^{\infty}\left(\mathbb{R}_{x}^{n}\right)} \leqq C_{11}\left|\partial_{z}^{\alpha} f(z)\right|_{L^{2}\left(\mathbb{R}_{x}^{n}\right)}^{1-b}\left(\sum_{|\beta|=m}\left|\partial_{x}^{\beta} \partial_{z}^{\alpha} f(z)\right|_{L^{2}\left(\mathbb{R}_{x}^{n}\right)}^{2}\right)^{b / 2}
$$


and

$$
\begin{aligned}
\left|\partial_{z}^{\alpha} f(z)\right|_{L^{\infty}\left(\mathbb{R}_{x}^{n}\right)}= & \left|e^{-i|x|^{2} / 2 t} \partial_{z}^{\alpha} f(z)\right|_{L^{\infty}\left(\mathbb{R}_{x}^{n}\right)} \leqq C_{12}|t|^{-n / 2}\left|\partial_{z}^{\alpha} f(z)\right|_{L^{2}\left(\mathbb{R}_{x}^{n}\right)}^{1-b} \\
& \cdot\left\{\begin{array}{l}
\left(\sum_{|\beta|=m}\left|J_{x}^{\beta} \partial_{z}^{\alpha} f(z)\right|_{L^{2}\left(\mathbb{R}_{x}^{n}\right)}^{2}\right)^{b / 2} \\
\left(\sum_{|\beta|=m}\left|\bar{J}_{x}^{\beta} \partial_{z}^{\alpha} f(z)\right|_{L^{2}\left(\mathbb{R}_{x}^{n}\right)}^{2}\right)^{b / 2}
\end{array}\right.
\end{aligned}
$$

In the same way as in the proof of (2.8) in [6], we have for $g(z) \in A H^{n / 2,2}(r)$,

$$
\begin{aligned}
|g(z)|_{L^{2}\left(\mathbb{R}_{x}^{n}\right)}^{2} & \leqq 2^{n} \sum_{\gamma} \frac{(2 y)^{2 \gamma}}{2 \gamma !}\left|\partial_{x}^{\gamma} g(x)\right|_{L^{2}\left(\mathbb{R}_{x}^{n}\right)}^{2} \\
& =2^{n} \int_{\mathbb{R}_{\xi}^{n}} \prod_{j=1}^{n} \cosh 2 y_{j} \xi_{j}|\hat{g}(\xi)|^{2} d \xi .
\end{aligned}
$$

Since

$$
\cosh 2 y_{j} \xi_{j} \leqq 1+\sinh 2\left|y_{j}\right|\left|\xi_{j}\right|
$$

from (3.7) and Lemma 1 it follows that with $C_{13}=(2 \sqrt{2})^{n}$,

$$
\begin{aligned}
&|g(z)|_{L^{2}\left(\mathbb{R}_{x}^{n}\right)}^{2} \leqq 2^{n} \int_{R_{\xi}^{n}} \prod_{j=1}^{n}\left(1+\sinh 2\left|y_{j}\right|\left|\xi_{j}\right|\right)|\hat{g}(\xi)|^{2} d \xi \\
& \leqq 2^{n} \int_{\mathbb{R}_{\xi}^{n}} \prod_{j=1}^{n} \frac{\sinh 2 r\left|\xi_{j}\right|}{2 r\left|\xi_{j}\right|}\left(1+2 r\left|\xi_{j}\right|\right)|\hat{g}(\xi)|^{2} d \xi \\
& \leqq C_{13} \int_{\mathbb{R}_{\xi}^{n}} \prod_{j=1}^{n} \frac{\sinh 2 r\left|\xi_{j}\right|}{2 r\left|\xi_{j}\right|}\left|\left(1+4 r^{2} \xi_{j}^{2}\right)^{1 / 4} \hat{g}(\xi)\right|^{2} d \xi \\
&=C_{13} \int_{\mathbb{R}_{\xi}^{n}} \prod_{j=1}^{n} \sum_{\gamma_{j}=0}^{\infty} \frac{(2 r)^{2 \gamma_{j}}}{\left(2 \gamma_{j}+1\right) !}\left|\xi_{j}^{\gamma_{j}}\left(1+4 r^{2} \xi_{j}^{2}\right)^{1 / 4} \hat{g}(\xi)\right|^{2} d \xi \\
& \leqq C_{13} \int_{\mathbb{R}_{\xi}^{n}} \prod_{j=1}^{n} \sum_{\gamma_{j}=0}^{\infty} Q\left(\gamma_{j}, r\right)\left|\xi_{j}^{\gamma_{j}} \prod_{j=1}^{n}\left(1+4 r^{2} \xi_{j}^{2}\right)^{1 / 4} \hat{g}(\xi)\right|^{2} d \xi \\
&=C_{13} \sum_{\gamma} Q(\gamma, r)\left|\partial_{x}^{\gamma}\left[\mathscr{F}^{-1} \prod_{j=1}^{n}\left(1+4 r^{2} \xi_{j}^{2}\right)^{1 / 4} \mathscr{F} g\right](x)\right|_{L^{2}\left(\mathbb{R}_{x}^{n}\right)}^{2} \\
&=C_{13}\left|P\left(2 i r \partial_{x}\right) g(z)\right|_{A L^{2}(r)}^{2}
\end{aligned}
$$

We have by (3.5), (3.8) and the equality $\partial_{x}^{\beta} \partial_{z}^{\alpha} f(z)=\partial_{z}^{\alpha+\beta} f(z)$,

$$
\begin{aligned}
\left|\partial_{z}^{\alpha} f(z)\right|_{L^{\infty}\left(\mathbb{R}_{x}^{n}\right) \leqq} & C_{14}\left|P\left(2 i r \partial_{x}\right) \partial_{z}^{\alpha} f(z)\right|_{A L^{2}(r)}^{1-b} \\
& \cdot\left(\sum_{|\beta|=m}\left|P\left(2 i r \partial_{x}\right) \partial_{z}^{\alpha+\beta} f(z)\right|_{A L^{2}(r)}^{2}\right)^{b / 2} .
\end{aligned}
$$

This shows (3.1). We have by the Taylor expansion and Lemma A (see Appendix)

$$
J_{x}^{\beta} \partial_{z}^{\alpha} f(z)=J_{x}^{\beta} \sum_{\gamma} \frac{(i y)^{\gamma}}{\gamma !} \partial_{x}^{\gamma+\alpha} f(x)=\sum_{\gamma} \frac{(i y)^{\gamma}}{\gamma !} \partial_{x}^{\gamma} L_{x}(\alpha, \beta) f(x) .
$$


In the same way as in the proof of (2.8) in [6],

$$
\left|J_{x}^{\beta} \partial_{z}^{\alpha} f(z)\right|_{L^{2}\left(\mathbb{B}_{x}^{n}\right)}^{2} \leqq 2^{n} \sum_{\gamma} \frac{(2 y)^{2 \gamma}}{2 \gamma !}\left|\partial_{x}^{\gamma} L_{x}(\alpha, \beta) f(x)\right|_{L^{2}\left(\mathbb{R}_{x}^{n}\right)}^{2} .
$$

By virtue of (3.9), (3.7) and (3.8),

$$
\left|J_{x}^{\beta} \partial_{z}^{\alpha} f(z)\right|_{L^{2}\left(\mathbb{R}_{x}^{n}\right)}^{2} \leqq(4 \sqrt{2})^{n}\left|P\left(2 i r \partial_{x}\right) L_{z}(\alpha, \beta) f(z)\right|_{A L^{2}(r)}^{2} .
$$

Similarly we have

$$
\left|\bar{J}_{x}^{\beta} \partial_{z}^{\alpha} f(z)\right|_{L^{2}\left(\mathbb{R}_{x}^{n}\right)}^{2} \leqq(4 \sqrt{2})^{n}\left|P\left(2 i r \partial_{x}\right) \overline{L_{\bar{z}}}(\alpha, \beta) f(z)\right|_{A L^{2}(r)}^{2},
$$

where $\bar{L}_{x}(\alpha, \beta)=\bar{L}_{x_{1}}\left(\alpha_{1}, \beta_{1}\right) \cdots \bar{L}_{x_{n}}\left(\alpha_{n}, \beta_{n}\right)$ is the complex conjugate of $L_{x}(\alpha, \beta)$, namely,

$$
\bar{L}_{x_{j}}\left(\alpha_{j}, \beta_{j}\right)=\sum_{\delta_{j}=0}^{\beta_{j}} \sum_{v_{j}=0}^{\min \left(\beta_{j}-\delta_{j}, \alpha_{j}\right)}\left(\begin{array}{c}
\beta_{j} \\
\delta_{j}
\end{array}\right)\left(\begin{array}{c}
\beta_{j}-\delta_{j} \\
v_{j}
\end{array}\right)\left(\begin{array}{c}
\alpha_{j} \\
v_{j}
\end{array}\right)(-1)^{v_{j}} v_{j} ! \partial_{x_{j}}^{\alpha_{j}-v_{j}}\left(i y_{j}\right)^{\delta_{J}} \bar{J}_{x_{j}}^{\beta_{j}-\delta_{j}-v_{j}} .
$$

We easily see that by (3.10) and (3.11)

$$
\sum_{|\beta|=m}\left|J_{x}^{\beta} \partial_{z}^{\alpha} f(z)\right|_{L^{2}\left(\mathbb{R}_{x}^{n}\right)}^{2} \leqq C_{15}(\alpha) \sum_{\substack{\delta \leqq \beta \\|\beta|=m}} \sum_{v \leqq \min (\beta-\delta, \alpha)}\left|P\left(2 i r \partial_{x}\right)(-i y)^{\delta} \partial_{z}^{\alpha-v} J_{z}^{\beta-\delta-v} f(z)\right|_{A L^{2}(r)}^{2}
$$

and

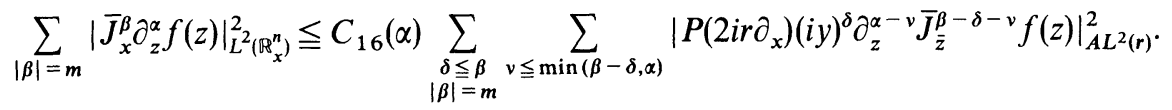

From (3.6), (3.8), (3.12) and (3.13) it follows that

$$
\left|\partial_{z}^{\alpha} f(z)\right|_{L^{\infty}\left(\mathbb{R}_{x}^{n}\right)} \leqq \text { the right-hand side of (3.2). }
$$

The inequalities (3.2) follow from the above immediately.

We next prove (3.3). We put $f(x)=e^{-i|x|^{2} / 2 t} g(x)$ and $r=|t|$ in (3.1). Then we have

$$
\begin{aligned}
\left|\left(i t \partial_{z}\right)^{\alpha} e^{-i|z|^{2} / 2 t} g(z)\right|_{A L^{x}(|t|)} \leqq & C_{9}|t|^{-n / 2}\left|P\left(2 i t \partial_{x}\right)\left(i t \partial_{z}\right)^{\alpha} e^{-i|z|^{2} / 2 t} g(z)\right|_{A L^{2}(|t|)}^{1-b} \\
& \cdot\left(\sum_{|\beta|=m}\left|P\left(2 i t \partial_{x}\right)\left(i t \partial_{z}\right)^{\alpha+\beta} e^{-i|z|^{2} / 2 t} g(z)\right|_{A L^{2}(|t|)}^{2}\right)^{b / 2} .
\end{aligned}
$$

By Lemma 1 and a simple calculation (see Remark 2) we get

$$
\begin{aligned}
\left|P\left(2 i t \partial_{x}\right)\left(i t \partial_{z}\right)^{\alpha} e^{-i|z|^{2} / 2 t} g(z)\right|_{A L^{2}(|t|)}^{2} & =\sum_{\gamma} Q(\gamma,|t|)\left|\partial_{x}^{\gamma} P\left(2 i t \partial_{x}\right)\left(i t \partial_{x}\right)^{\alpha} e^{-i|x|^{2} / 2 t} g(x)\right|_{L^{2}\left(\mathbb{R}_{x}^{n}\right)}^{2} \\
& =\sum_{\gamma} Q(\gamma, 1)\left|e^{i|x|^{2} / 2 t} P\left(2 i t \partial_{x}\right)\left(i t \partial_{x}\right)^{\gamma+\alpha} e^{-i|x|^{2} / 2 t} g(x)\right|_{L^{2}\left(\mathbb{R}_{x}^{n}\right)}^{2} \\
& =\sum_{\gamma} Q(\gamma, 1)\left|U(t) P(2 x) x^{\gamma+\alpha} U(-t) g(x)\right|_{L^{2}\left(\mathbb{R}_{x}^{n}\right)^{*}}^{2}
\end{aligned}
$$


The right-hand side of $(3.15)=\sum_{\gamma} Q(\gamma, 1)\left|P\left(2 i \partial_{\xi}\right) \partial_{\xi}^{\gamma+\alpha}[\mathscr{F} U(-t) g](\xi)\right|_{L^{2}\left(\mathbb{R}_{\xi}^{n}\right)}^{2}$

$$
=\sum_{\gamma} Q(\gamma, 1)\left|\partial_{\xi}^{\gamma} P\left(2 i \partial_{\xi}\right) \partial_{\xi}^{\alpha} e^{i t|\xi|^{2} / 2} \hat{g}(\xi)\right|_{L^{2}\left(L_{\xi}^{n}\right)}^{2}
$$

Thus we have by (3.15), (3.16) and Lemma 1,

$$
\left|P\left(2 i t \partial_{x}\right)\left(i t \partial_{z}\right)^{\alpha} e^{-i|z|^{2} / 2 t} g(z)\right|_{A L^{2}(|t|)}^{2}=\left|P\left(2 i \partial_{x}\right) \partial_{z}^{\alpha} e^{i t|z|^{2} / 2} \hat{g}(z)\right|_{A L^{2}(1)}^{2} .
$$

By applying (3.17) to (3.14), we obtain (3.3). We finally prove (3.4). In the same way as in the proof of (3.3), we put $f(x)=e^{-i|x|^{2} / 2 t} g(x)$ and $r=|t|$ in (3.2b). Then we have

$$
\begin{aligned}
\left|\left(i t \partial_{z}\right)^{\alpha} e^{-i|z|^{2} / 2 t} g(z)\right|_{A L^{x}(|t|)} \leqq & C_{10}(\alpha)|t|^{-n / 2}\left|P\left(2 i t \partial_{x}\right)\left(i t \partial_{z}\right)^{\alpha} e^{-i|z|^{2} / 2 t} g(z)\right|_{A L^{2}(|t|)}^{1-b} \\
& \cdot \sum_{\substack{\delta \leqq \beta \\
|\beta|=m}} \sum_{v \leqq \min (\beta-\delta, \alpha)}\left(|t|^{2|\delta|} \mid P\left(2 i t \partial_{x}\right)\left(i t \partial_{z}\right)^{\alpha-v}(i t)^{|v|}\right. \\
& \left.\left.\cdot \bar{J}_{\bar{z}}^{\beta-\delta-v-v} e^{-i|z|^{2} / 2 t} g(z)\right|_{A L^{2}(|t|)} ^{2}\right)^{b / 2}
\end{aligned}
$$

By a direct calculation we have

$$
\begin{aligned}
& |t|^{2|\delta|}\left|P\left(2 i t \partial_{x}\right)\left(i t \partial_{z}\right)^{\alpha-v}(i t)^{|v|} \bar{J}_{\bar{z}}^{\beta-\delta-v} e^{-i|z|^{2} / 2 t} g(z)\right|_{A L^{2}(|t|)}^{2} \\
& \quad=|t|^{2(|\delta|+|v|)}\left|P\left(2 i t \partial_{x}\right)\left(i t \partial_{z}\right)^{\alpha-v} e^{-i|z|^{2} / 2 t}\left(-i t \partial_{z}\right)^{\beta-\delta-v} g(z)\right|_{A L^{2}(t \mid)}^{2} \\
& \quad=|t|^{2|\beta|}\left|P\left(2 i t \partial_{x}\right)\left(i t \partial_{z}\right)^{\alpha-v} e^{-i|z|^{2} / 2 t} \partial_{z}^{\beta-\delta-v} g(z)\right|_{A L^{2}(|t|)}^{2}
\end{aligned}
$$

In the same way as in the proof of (3.17),

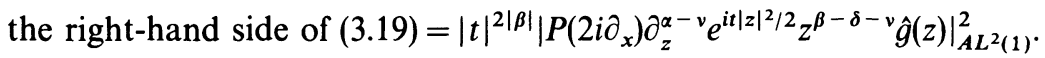

By (3.18), (3.20) and (3.17) we have (3.4). Q.E.D.

The next estimates concerning the nonlinear term $|f(x)|^{2} f(x)$ are obtained by using Lemma 3.1 and gauge invariance which the nonlinear term satisfies. It is clear that $|f(x)|^{2} f(x)$ satisfies gauge invariance since $|\omega f(x)|^{2} \omega f(x)=\omega\left(|f(x)|^{2} f(x)\right)$ for any complex number $\omega$ with $|\omega|=1$. We note that if $f(z)$ is analytic on $S(r)$ and $f(z) \in A L^{p}(r)$, then so is $\overline{f(\bar{z})}$ and $|f(z)|_{A L^{p}(r)}=\mid \overline{f(\bar{z})}_{A L^{p}(r)}$. The restriction of $\overline{f(\bar{z})}$ to the real axis coincides with $\overline{f(x)}$, and hence $\overline{f(\bar{z})} f^{2}(z)$ is an analytic continuation of $|f(x)|^{2} f(x)$.

Lemma 3.2. 1. Let $J_{z}^{\beta} f(z), J_{z}^{\beta} g(z) \in A H^{|\alpha|, 2}(r)$ for $|\alpha|+|\beta| \leqq 2 n+1$. Then we have

$$
\begin{gathered}
\left|\overline{f(\bar{z})} f^{2}(z)\right|_{B_{1}(t)} \leqq C_{17} \cdot(1+|t|)^{-n}|f(z)|_{B_{1}(t)}^{3}, \\
\left|\overline{f(\bar{z})} f^{2}(z)-\overline{g(\bar{z})} g^{2}(z)\right|_{B_{1}(t)} \leqq C_{18} \cdot(1+|t|)^{-n}\left(|f(z)|_{B_{1}(t)}+|g(z)|_{B_{1}(t)}\right)^{2}|f(z)-g(z)|_{B_{1}(t)} .
\end{gathered}
$$

2. We assume that $e^{i t|\xi|^{2} / 2} \xi^{\beta} \hat{f}(\xi), e^{i t|\xi|^{2} / 2} \xi^{\beta} \hat{g}(\xi)$ have analytic continuations to $S(1)$ and $e^{i t|z|^{2} / 2} z^{\beta} \hat{f}(z), e^{i t|z|^{2} / 2} z^{\beta} \hat{g}(z) \in A H^{|\alpha|, 2}(1)$ for $|\alpha|+|\beta| \leqq 2 n+1$. Then we have

$$
\begin{gathered}
\left|\overline{f(\bar{z})} f^{2}(z)\right|_{B_{2}(t)} \leqq C_{19} \cdot(1+|t|)^{-n}|f(z)|_{B_{2}(t)}^{3}, \\
\left|\overline{f(\bar{z})} f^{2}(z)-\overline{g(\bar{z})} g^{2}(z)\right|_{B_{2}(t)} \leqq C_{20} \cdot(1+|t|)^{-n}\left(|f(z)|_{B_{2}(t)}+|g(z)|_{B_{2}(t)}\right)^{2}|f(z)-g(z)|_{B_{2}(t)} .
\end{gathered}
$$


Proof. By gauge invariance of $|f(x)|^{2} f(x)$ and a simple calculation

$$
\left|\overline{f(\bar{z})} f^{2}(z)\right|_{B_{1}(t)}^{2} \leqq C_{21} \sum_{\begin{array}{c}
|\alpha|=|\gamma|+|\delta|+|\mu| \\
|\beta|=|\nu|+|\tau|+|\omega| \\
|\alpha|+|\beta| \leqq 2 n+1
\end{array}} \mid \partial_{z}^{\gamma} J_{z}^{v} f(z) \cdot \partial_{z}^{\delta} J_{z}^{\tau} f(z) \cdot \partial_{z}^{\mu} \bar{J}_{\bar{z}}^{\omega} \overline{\left.f(\bar{z})\right|_{A L^{2}(r)} ^{2} .}
$$

If $a, b, c \in \mathbb{N}$ and $a+b+c \leqq 2 n+1$, then at least two of them are less than or equal to $n$. Therefore we have by the above inequality

$$
\left|\overline{f(\bar{z})} f^{2}(z)\right|_{B_{1}(t)}^{2} \leqq C_{22} \sum_{|\alpha|+|\beta| \leqq n}\left|\partial_{z}^{\alpha} J_{z}^{\beta} f(z)\right|_{A L^{\infty}(r)}^{4}|f(z)|_{B_{1}(t)}^{2},
$$

where we have used the fact that

$$
\left|\partial_{z}^{\mu} \overline{J_{\bar{z}}^{\omega}} \overline{f(\bar{z})}\right|_{A L^{p}(r)}=\left|\partial_{z}^{\mu} J_{z}^{\omega} f(z)\right|_{A L^{p}(r)} .
$$

By Lemma 3.1 ((3.1),(3.2a)),

$$
\begin{aligned}
& \left|\partial_{z}^{\alpha} J_{z}^{\beta} f(z)\right|_{A L^{\alpha}(r)}^{4} \leqq C_{23} \cdot(1+|t|)^{-2 n}\left|P\left(2 i r \partial_{x}\right) \partial_{z}^{\alpha} J_{z}^{\beta} f(z)\right|_{A L^{2}(r)}^{4(1-b)} \\
& \cdot\left(\sum_{|y|=[n / 2]+1}\left|P\left(2 i r \partial_{x}\right) \partial_{z}^{\alpha+\gamma} J_{z}^{\beta} f(z)\right|_{A L^{2}(r)}^{2}\right. \\
& \left.+\sum_{\substack{\delta \leq y \\
|\gamma|=[n / 2]+1}} \sum_{\nu \leqq \min (\gamma-\delta, \alpha)} r^{2|\delta|}\left|P\left(2 i r \partial_{x}\right) \partial_{z}^{\alpha-v} J_{z}^{\beta+\gamma-\delta-v} f(z)\right|_{A L^{2}(r)}^{2}\right)^{2 b}
\end{aligned}
$$

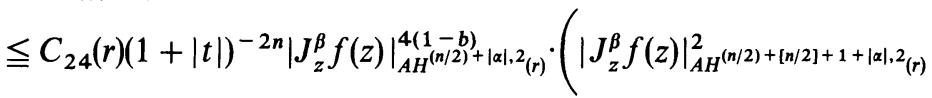

$$
\begin{aligned}
& \left.+\sum_{\substack{\delta \leqq \gamma \\
|\gamma|=[n / 2]+1}} \sum_{\nu \leqq \min (\gamma-\delta, \alpha)}\left|J_{z}^{\beta+\gamma-\delta-v} f(z)\right|_{A H^{(n / 2)+|\alpha-v|, 2}(r)}^{2}\right)^{2 b} .
\end{aligned}
$$

Since $(n / 2)+[n / 2]+1+|\alpha|+|\beta| \leqq 2 n+1$, we have by (3.24)

$$
\sum_{|\alpha|+|\beta| \leqq n}\left|\partial_{z}^{\alpha} J_{z}^{\beta} f(z)\right|_{A L^{\infty}(r)}^{4} \leqq C_{25}(r)(1+|t|)^{-2 n}|f(z)|_{B_{1}(t)}^{4}
$$

From (3.25) and (3.23), (3.21) follows. We have

$$
\begin{aligned}
\overline{f(\bar{z})} f^{2}(z)-\overline{g(\bar{z})} g^{2}(z) & =\overline{f(\bar{z})}\left(f^{2}(z)-g^{2}(z)\right)+\left(\overline{f(\bar{z})}-\overline{g(\bar{z}))} g^{2}(z)\right. \\
& =\overline{f(\bar{z})}(f(z)-g(z))(f(z)+g(z))+\overline{(f(\bar{z}))}-\overline{g(\bar{z}))} g^{2}(z) .
\end{aligned}
$$

In the same way as in the proof of (3.21), from (3.26) we obtain (3.22) immediately.

We next prove $\left(3.21^{\prime}\right)$. By the same argument as in Remark 2 we have

$$
\left|\overline{f(\bar{z})} f^{2}(z)\right|_{B_{2}(t)}^{2}=\sum_{|\alpha|+|\beta| \leqq 2 n+1}\left|\left(i t \partial_{z}\right)^{\alpha} e^{-i|z|^{2} / 2 t} \partial_{z}^{\beta} \overline{f(\bar{z})} f^{2}(z)\right|_{A L^{2}(|t|)}^{2}
$$

By a direct calculation, the right-hand side of (3.27) is dominated by

$$
\begin{aligned}
& C_{26} \sum_{\substack{|\alpha|=|\gamma|+|\delta|+|\mu| \\
|\beta|=|y|+|\tau|+|\omega| \\
|\alpha|+|\beta| \leqq 2 n+1}} \mid\left(i t \partial_{z}\right)^{\gamma} e^{-i|z|^{2} / 2 t} \partial_{z}^{v} f(z) \cdot\left(i t \partial_{z}\right)^{\delta} e^{-i|z|^{2} / 2 t} \partial_{z}^{\tau} f(z) \\
& \left.\cdot\left(i t \partial_{z}\right)^{\mu} e^{\left.i|z|\right|^{2} / 2 t} \partial_{z}^{\omega} \overline{f(\bar{z})}\right|_{A L^{2}(|t|)} ^{2} .
\end{aligned}
$$


Therefore we obtain in the same way as in the proof of (3.23),

$$
\left|\overline{f(\bar{z})} f^{2}(z)\right|_{B_{2}}^{2} \leqq C_{27} \sum_{|\alpha|+|\beta| \leqq n}\left|\left(i t \partial_{z}\right)^{\alpha} e^{-i|z|^{2} / 2 t} \partial_{z}^{\beta} f(z)\right|_{A L^{\infty}(|t|)}^{4}|f(z)|_{B_{2}(t)}^{2}
$$

By Lemma 3.1 ((3.3), (3.4))

$$
\begin{aligned}
& \left|\left(i t \partial_{z}\right)^{\alpha} e^{-i|z|^{2} / 2 t} \partial_{z}^{\beta} f(z)\right|_{A L^{\infty}(|t|)}^{4} \\
& \leqq C_{28} \cdot(1+|t|)^{-2 n}\left|P\left(2 i \partial_{x}\right) \partial_{z}^{\alpha} e^{i t|z|^{2} / 2} z^{\beta} \hat{f}(z)\right|_{A L^{2}(1)}^{4(1-b)} \\
& \cdot\left(\sum_{|\gamma|=[n / 2]+1}\left|P\left(2 i \partial_{x}\right) \partial_{z}^{\alpha+\gamma} e^{i t|z|^{2} / 2} z^{\beta} \widehat{f}(z)\right|_{A L^{2}(1)}^{2}\right. \\
& \left.+\sum_{\substack{\delta \leqq \gamma \\
|\gamma|=[n / 2]+1}} \sum_{v \leqq \min (\gamma-\delta, \alpha)}\left|P\left(2 i \partial_{x}\right) \partial_{z}^{\alpha-v} e^{i t|z|^{2} / 2} z^{\beta+\gamma-\delta-v} \hat{f}(z)\right|_{A L^{2}(1)}^{2}\right)^{2 b} \\
& \leqq C_{29} \cdot(1+|t|)^{-2 n}\left|e^{i t|z|^{2} / 2} z^{\beta} \hat{f}(z)\right|_{A H^{(n / 2)+|\alpha|, 2(1)}}^{4(1-b)} \cdot\left(\left|e^{i t|z|^{2} / 2} z^{\beta} \hat{f}(z)\right|_{A H^{(n / 2)+[n / 2]+1+|\alpha|, 2}(1)}^{2}\right. \\
& \left.+\sum_{\substack{\delta \leqq \gamma \\
|\gamma|=[n / 2]+1}} \sum_{\nu \leqq \min (\gamma-\delta, \alpha)}\left|e^{i t|z|^{2} / 2 z \beta+\gamma-\delta-v} \hat{f}(z)\right|_{A H^{(n / 2)+\mid \alpha-v, 2}}^{2}\right)^{2 b}
\end{aligned}
$$

Since $(n / 2)+[n / 2]+1+|\alpha|+|\beta| \leqq 2 n+1$, we have by (3.29),

$$
\sum_{|\alpha|+|\beta| \leqq n}\left|\left(i t \partial_{z}\right)^{\alpha} e^{-i|z|^{2} / 2 t} \partial_{z}^{\beta} f(z)\right|_{A L^{\infty}(|t|)}^{4} \leqq C_{30} \cdot(1+|t|)^{-2 n}|f(z)|_{B_{2}(t)}^{4} .
$$

From (3.28) and (3.30), (3.21') follows. In the same way as in the proof of (3.22), we have $\left(3.22^{\prime}\right)$ by using the proof of $\left(3.21^{\prime}\right)$, and so we omit it. Q.E.D.

Remark 4. We can treat more general nonlinear Schrödinger equations

$$
\begin{aligned}
i \partial_{t} u+\frac{1}{2} \Delta u & =\lambda|u|^{2 p} u, & (t, x) & \in \mathbb{R} \times \mathbb{R}_{x}^{n}, \\
u(0, x) & =\phi(x), & & x \in \mathbb{R}_{x}^{n},
\end{aligned}
$$

where $\lambda \in \mathbb{R}, p \in \mathbb{N}$ for $n \geqq 2, p \in \mathbb{N}$ and $p \geqq 2$ for $n=1$. Moreover if we restrict our attention to the local existence theorem of analytic solutions, our method is applicable to the nonlinear Klein-Gordon equations,

$$
\begin{gathered}
\partial_{t t} u-\Delta u+u=f(u), \quad(t, x) \in \mathbb{R} \times \mathbb{R}_{x}^{n}, \\
u(0, x)=\phi_{1}(x), \quad \partial_{t} u(0, x)=\phi_{2}(x), \quad x \in \mathbb{R}_{x}^{n},
\end{gathered}
$$

where $f(\lambda)$ is a real analytic function of $\lambda, \phi_{1}(x)$ and $\phi_{2}(x)$ have analytic continuations to $S(r)$ and $\phi_{1}(z) \in A H^{2 n+1,2}(r)$ and $\phi_{2}(z) \in A H^{2 n, 2}(r)$, respectively.

\section{Appendix}

Lemma A. We let $L_{x}(\alpha, \beta)=L_{x_{1}}\left(\alpha_{1}, \beta_{1}\right) \cdots L_{x_{n}}\left(\alpha_{n}, \beta_{n}\right)$, where $L_{x_{j}}\left(\alpha_{j}, \beta_{j}\right)=\sum_{\delta_{j}=0}^{\beta_{j}} \sum_{v_{j}=0}^{\min \left(\beta_{j}-\delta_{j}, \alpha_{j}\right)}\left(\begin{array}{c}\beta_{j} \\ \delta_{j}\end{array}\right)\left(\begin{array}{c}\beta_{j}-\delta_{j} \\ v_{j}\end{array}\right)\left(\begin{array}{c}\alpha_{j} \\ v_{j}\end{array}\right)(-1)^{v_{j}} v_{j} ! \partial_{x_{j}}^{\alpha_{j}-v_{j}}\left(-i y_{j}\right)^{\delta_{j}} J_{x_{j}}^{\beta_{j}-\delta_{j}-v_{j}}$ 
Then we have for any $L_{x}(\alpha, \beta) f(z) \in A L^{2}(r)$,

$$
\sum_{\gamma} \frac{(i y)^{\gamma}}{\gamma !} J_{x}^{\beta} \partial_{x}^{\gamma+\alpha} f(x)=\sum_{\gamma} \frac{(i y)^{\gamma}}{\gamma !} \partial_{x}^{\gamma} L_{x}(\alpha, \beta) f(x) .
$$

Proof. Since $\left[J_{x_{j}}, \partial_{x_{k}}\right]=0$ if $j \neq k$, we have

$$
J_{x}^{\beta} \partial_{x}^{\gamma+\alpha} f(x)=\prod_{j=1}^{n} J_{x_{j}}^{\beta_{j}} \partial_{x_{j}}^{\gamma_{j}+\alpha_{j}} f(x)
$$

If we can prove that

$$
\sum_{\gamma_{j}=0}^{\infty} \frac{\left(i y_{j}\right)^{\gamma_{j}}}{\gamma_{j} !} J_{x_{j}}^{\beta_{j}} \partial_{x_{j}}^{\gamma_{j}+\alpha_{j}} f(x)=\sum_{\gamma_{j}=0}^{\infty} \frac{\left(i y_{j}\right)^{\gamma_{j}}}{\gamma_{j} !} \partial_{x_{j}}^{\gamma_{j}} L_{x_{j}}\left(\alpha_{j}, \beta_{j}\right) f(x),
$$

then the desired equality follows from (A.1) and the fact that

$$
\left[\partial_{x_{j}}^{\gamma_{j}}, L_{x_{k}}\left(\alpha_{k}, \beta_{k}\right)\right]=0 \text { if } j \neq k \text {. }
$$

We will show (A.2). For simplicity we drop the index $j$. By a direct calculation

$$
\begin{aligned}
{\left[J_{x}^{\beta}, \partial_{x}^{\gamma+\alpha}\right] } & =\sum_{\delta=1}^{\beta}\left(\begin{array}{l}
\beta \\
\delta
\end{array}\right)\left[J_{x},\left[J_{x}, \ldots,[J_{x}, \partial_{x}^{\gamma} \overbrace{] \cdots]}^{\delta \text {-times }} J_{x}^{\beta-\delta}\right.\right. \\
& = \begin{cases}\sum_{\delta=0}^{\beta}\left(\begin{array}{l}
\beta \\
\delta
\end{array}\right)\left(\begin{array}{l}
\gamma \\
\delta
\end{array}\right)(-1)^{\delta} \delta ! \partial_{x}^{\gamma-\delta} J_{x}^{\beta-\delta} & \text { for } \gamma \geqq \delta . \\
0, & \text { for } \gamma<\delta .\end{cases}
\end{aligned}
$$

Thus we have by the above equality

$$
J_{x}^{\beta} \partial_{x}^{\gamma} f(x)=\sum_{\delta=0}^{\min (\beta, \gamma)}\left(\begin{array}{l}
\beta \\
\delta
\end{array}\right)\left(\begin{array}{l}
\gamma \\
\delta
\end{array}\right)(-1)^{\delta} \delta ! \partial_{x}^{\gamma-\delta} J_{x}^{\beta-\delta} f(x) .
$$

By using (A.3) we get

$$
\begin{aligned}
\sum_{\gamma=0}^{\beta} \frac{(i y)^{\gamma}}{\gamma !} J_{x}^{\beta} \partial_{x}^{\gamma} \partial_{x}^{\alpha} f(x) & =\sum_{\gamma=0}^{\infty} \frac{(i y)^{\gamma}}{\gamma !} \sum_{\delta=0}^{\min (\beta, \gamma)} \frac{\gamma !}{(\gamma-\delta) !}(-1)^{\delta}\left(\begin{array}{l}
\beta \\
\delta
\end{array}\right) \partial_{x}^{\gamma-\delta} J_{x}^{\beta-\delta} \partial_{x}^{\alpha} f(x) \\
& =\sum_{\gamma=0}^{\infty} \sum_{\delta=0}^{\min (\beta, \gamma)}\left(\frac{(i y)^{\gamma}}{(\gamma-\delta) !}(-1)^{\delta}\left(\begin{array}{l}
\beta \\
\delta
\end{array}\right) \partial_{x}^{\gamma-\delta} J_{x}^{\beta-\delta} \partial_{x}^{\alpha} f(x)\right) \\
& =\sum_{\delta=0}^{\beta} \sum_{\gamma=\delta}^{\infty} \frac{(i y)^{\gamma-\delta}}{(\gamma-\delta) !} \partial_{x}^{\gamma-\delta}(-i y)^{\delta}\left(\begin{array}{l}
\beta \\
\delta
\end{array}\right) J_{x}^{\beta-\delta} \partial_{x}^{\alpha} f(x) \\
& =\sum_{\gamma=0}^{\infty} \frac{(i y)^{\gamma}}{\gamma !} \partial_{x}^{\gamma}\left(\sum_{\delta=0}^{\beta}(-i y)^{\delta}\left(\begin{array}{l}
\beta \\
\delta
\end{array}\right) J_{x}^{\beta-\delta} \partial_{x}^{\alpha} f(x)\right) .
\end{aligned}
$$

We again use (A.3) to obtain

$$
J_{x}^{\beta-\delta} \partial_{x}^{\alpha} f(x)=\sum_{v=0}^{\min (\beta-\delta, \alpha)}\left(\begin{array}{c}
\beta-\delta \\
v
\end{array}\right)\left(\begin{array}{l}
\alpha \\
v
\end{array}\right)(-1)^{v} v ! \partial_{x}^{\alpha-v} J_{x}^{\beta-\delta-v} f(x) .
$$


By (A.4) and (A.5)

$$
\begin{aligned}
\sum_{\gamma=0}^{\infty} & \frac{(i y)^{\gamma}}{\gamma !} J_{x}^{\beta} \partial_{x}^{\gamma+\alpha} f(x) \\
& =\sum_{\gamma=0}^{\infty} \frac{(i y)^{\gamma}}{\gamma !} \partial_{x}^{\gamma}\left(\sum_{\delta=0}^{\beta} \sum_{v=0}^{\min (\beta-\delta, \alpha)}\left(\begin{array}{c}
\beta \\
\delta
\end{array}\right)\left(\begin{array}{c}
\beta-\delta \\
v
\end{array}\right)\left(\begin{array}{l}
\alpha \\
v
\end{array}\right)(-1)^{v} v ! \partial_{x}^{\alpha-v}(-i y)^{\delta} J_{x}^{\beta-\delta-v} f(x)\right) \\
& =\sum_{\gamma=0}^{\infty} \frac{(i y)^{\gamma}}{\gamma !} \partial_{x}^{\gamma} L_{x}(\alpha, \beta) f(x) .
\end{aligned}
$$

This shows (A.2). Q.E.D.

Acknowledgement. The authors would like to thank the referee for careful reading of the first manuscript and many valuable comments.

\section{References}

1. Aikawa, H., Hayashi, N., Saitoh, S.: Analyticity of solutions for semi-linear heat equations in one space dimension, submitted for publication

2. Baillon, J. B., Cazenave, T., Figueira, M.: Equation de Schrödinger nonlinéaire. C. R. Acad. Sci. Paris 284, 864-872 (1977)

3. Dong, C. C., Li, S.: On the initial value problem for a nonlinear Schrödinger equation. J. Diff. Equations 42, 335-365 (1981)

4. Ginibre, J., Velo, G.: On a class of nonlinear Schrödinger equation, I, II. J. Funct. Anal. 32, 1-32, 33-71 (1979); III. Ann. Inst. Henri Poincaré, Physique Théorique 28, 287-316 (1978)

5. Hayashi, N.: Classical solutions of nonlinear Schrödinger equations. Manuscript Math. 55, 171-190 (1986)

6. Hayashi, N., Saitoh, S.: Analyticity and smoothing effect for the Schrödinger equation. Ann. Inst. Henri Poincaré, Physique Théorique (to appear)

7. Hayashi, N., Nakamitsu, K., Tsutsumi, M.: On solutions of the initial value problem for the nonlinear Schrödinger equations. J. Funct. Anal. 71, 218-245 (1987)

8. Kato, T.: On nonlinear Schrödinger equations. Ann. Inst. Henri Poincaré, Physique Théorique 46, 113-129 (1987)

9. Kato, T., Masuda, K.: Nonlinear evolution equations and analyticity I. Ann. Inst. Henri Poincaré, Analyse non linéaire, 3, 455-467 (1986)

10. Klainerman, S.: Long-time behavior of solutions to nonlinear evolution equations. Arch. Rat. Mech. Anal. 28, 73-98 (1982)

11. Klainerman, S., Ponce, G.: Global, small amplitude solutions to nonlinear evolution equations. Commun. Pure Appl. Math. 36, 133-141 (1983)

12. Shatah, J.: Global existence of small solutions to nonlinear evolution equations. J. Diff. Equations 46, 409-425 (1982)

13. Simon, J. C. H., Taflin, E.: Wave operators and analytic solutions of non-linear Klein-Gordon equations and of non-linear Schrödinger equations. Commun. Math. Phys. 99, 541-562 (1985)

Communicated by B. Simon

Received May 5, 1989; in revised form September 27, 1989 
